\title{
TU/e EN⿴HONE

\section{Padé approximation of delays in cooperative ACC based on string stability requirements}

Citation for published version (APA):

Xing, H., Ploeg, J., \& Nijmeijer, H. (2016). Padé approximation of delays in cooperative ACC based on string stability requirements. IEEE Transactions on Intelligent Vehicles, 1(3), 277-286. [7839205].

https://doi.org/10.1109/TIV.2017.2662482

\section{Document license:}

TAVERNE

DOI:

10.1109/TIV.2017.2662482

Document status and date:

Published: 01/09/2016

\section{Document Version:}

Publisher's PDF, also known as Version of Record (includes final page, issue and volume numbers)

\section{Please check the document version of this publication:}

- A submitted manuscript is the version of the article upon submission and before peer-review. There can be important differences between the submitted version and the official published version of record. People interested in the research are advised to contact the author for the final version of the publication, or visit the $\mathrm{DOI}$ to the publisher's website.

- The final author version and the galley proof are versions of the publication after peer review.

- The final published version features the final layout of the paper including the volume, issue and page numbers.

Link to publication

\section{General rights}

Copyright and moral rights for the publications made accessible in the public portal are retained by the authors and/or other copyright owners and it is a condition of accessing publications that users recognise and abide by the legal requirements associated with these rights.

- Users may download and print one copy of any publication from the public portal for the purpose of private study or research.

- You may not further distribute the material or use it for any profit-making activity or commercial gain

- You may freely distribute the URL identifying the publication in the public portal.

If the publication is distributed under the terms of Article 25fa of the Dutch Copyright Act, indicated by the "Taverne" license above, please follow below link for the End User Agreement:

www.tue.nl/taverne

Take down policy

If you believe that this document breaches copyright please contact us at:

openaccess@tue.nl

providing details and we will investigate your claim. 


\title{
Padé Approximation of Delays in Cooperative ACC Based on String Stability Requirements
}

\author{
Haitao Xing, Jeroen Ploeg, and Henk Nijmeijer, Fellow, IEEE
}

\begin{abstract}
Cooperative adaptive cruise control (CACC) improves road throughput by employing intervehicle wireless communications. The inherent communication time delay and vehicle actuator delay significantly limit the minimum intervehicle distance in view of string stability requirements. Hence, controller design needs to consider both delays, which result in a nonrational transfer function representation of the CACC-controlled string. Padé approximations can be applied to arrive at a finitedimensional model, which allows for many standard control methods. Our objective is to provide a method to decide for the lowest possible order of the Padé approximation, which is sufficiently accurate in view of CACC (string) stability analysis. The constant time gap strategy and a one-vehicle look-ahead topology are adopted to develop a CACC stable string. First, based on the stable controller parameter region, a suitable order of Padé approximations of the vehicle actuator delay can been carried out in view of individual vehicle stability. Then, the minimum string-stable time gaps for a CACC system with both exact and approximated delays have been compared. The procedure with a Proportionalderivative controller to choose the approximation order of delays has been given, followed by the time-domain simulation validation.
\end{abstract}

Index Terms-Cooperative adaptive cruise control (CACC), Padé approximation, string stability, wireless communication delay.

\section{INTRODUCTION}

A DVANCED driver assistance systems have significantly developed in the last decades. Adaptive cruise control (ACC) systems are penetrating into the market. Equipped with radar or camera to detect the preceding vehicle, ACC systems relieve the drivers' task by automatically keeping a desired intervehicle distance [1]. To realize a shorter intervehicle distance and, consequently, to improve highway capacity, cooperative adaptive cruise control (CACC) systems, which use wireless intervehicle communications, have been developed [2], [3]. String stability, which refers to the attenuation of the effects of disturbances in upstream direction of the string of vehicles, is a primary requirement for a $\mathrm{CACC}$ string to prevent traffic jams while increasing highway throughput. Furthermore, string stability is

Manuscript received June 15, 2016; revised November 16, 2016; accepted January 21, 2017. Date of publication February 1, 2017; date of current version March 15, 2017. This work was supported by the China Scholarship Council under Grant 201306170017.

H. Xing and H. Nijmeijer are with the Eindhoven University of Technology, Mechanical Engineering Department, Eindhoven 5612 AZ, The Netherlands (e-mail: h.xing@tue.nl; h.nijmeijer@tue.nl).

J. Ploeg is with the TNO, Integrated Vehicle Safety Department, Helmond 5700 AT, The Netherlands (e-mail: jeroen.ploeg@tno.nl).

Digital Object Identifier 10.1109/TIV.2017.2662482 beneficial for safety and for fuel consumption particularly for commercial vehicles [4].

However, imperfections induced by the wireless communication, such as delays, sampling intervals, packet loss, and communication constraints, can affect string stability [5]. Thus, it is critical to take communication delays into account to design and analyze a CACC string.

The influence of communication delay on string stability has been well-known in the literature. In [6], for instance, the effects of communication delays from lead and preceding vehicles were analyzed through a partial fraction expansion approach. Naus et al. [2] derived a necessary and sufficient frequency-domain condition for string stability, considering communication delay. Ploeg et al. [7] presented theoretical analysis and experimental results, indicating that the existence of communication delay compromises string stability. In the Grand Cooperative Driving Challenge 2011 [8]-[11] the information of vehicles was communicated at $10 \mathrm{~Hz}$. In the GCDC 2016 [12], vehicle motion data update frequency was required to be $25 \mathrm{~Hz}$.

In a CACC string, besides wireless communication delays, there is also the vehicle actuator delay, which not only affects individual vehicle stability [13], but also has effects on string stability. From recent experiments on vehicle model validation [7], [14], there is an observable vehicle actuator delay value, which cannot be ignored. In [11], the vehicle actuator delay and actuator response lag were taken into account when designing CACC systems. Xiao et al. [15] presented a requirement for minimum time gap to guarantee string stability, considering parasitic vehicle delays and phase lags. Based on the Lyapunov-Razumikhin theorem, Huang and Ren [16] constructed a conservative upper bound for the vehicle actuator delay to guarantee individual vehicle stability, and derived a condition for string stability.

Hence, both the vehicle actuator delay and the wireless communication delay are of utmost importance in view of string stability in CACC systems.

However, considering time delays leads to a nonrational transfer function, which poses a problem for many controller design methods. To deal with the nonrational representation of time delays, some studies have paid attention to the applications of approximations for time delays. As an example, Morbidi et al. [17] analytically studied sufficient conditions for string stability with communication and actuator delays, while the delays were approximated with Maclaurin series expansions under the assumption that the product of the delay and the frequency was sufficiently small. However, Padé approximation is more frequently used for systems with time delays, since it often gives 
a better approximation of the delay system than the Maclaurin series of the same order, and it may still result in a suitable representation, whereas the Maclaurin series of the same order does not converge [18].

Padé approximations of time delays have been applied in CACC systems in some publications. In [11], a linear controller was designed, where vehicle actuator delay was approximated by a 2nd-order Padé approximation in a CACC string. In [19], a 4th-order Padé approximation was adopted to permit a finite-dimensional model of the vehicle dynamics in view of the vehicle response delay. In [20], both the vehicle and communication delays were described by a 3rd-order Padé approximation. Although a higher order Padé approximation leads to a more accurate representation, the resulting system is more complex. Thus, it is necessary to explicitly motivate a suitable order of the Padé approximation used in CACC systems. In addition, a too high-order Padé approximation may cause numerical problems, e.g., in [20], increasing the Padé approximation order for both delays by $n$ leads to an increment of the controller order by $2 n$, which will require an additional order-reduction step in the $\mathcal{H}_{\infty}$ controller synthesis to make the controller applicable in practice.

This paper focusses on a procedure to find a suitable (low) order for the approximation for the delays in view of both individual vehicle stability and string stability. To this end, first, to preserve a similar (individual) stability property of both the CACC system with approximated delays and the one with exact delays, both systems should have approximately the same range of controller parameters that lead to stability. Next, in view of string stability, the minimum time gap for the approximated CACC system with selected order should be close to the one of the original system, where a variety of vehicle model parameters and communication delays have to be considered.

Based on a particular Proportional-derivative (PD) controller, the selected order of the Padé approximation of the delays is possibly also suitable when designing other controllers for CACC systems, which has been illustrated with a comparison of the PD controller as applied in this paper and the $\mathcal{H}_{\infty}$ controller from [20]. Thus, in CACC systems, the method proposed in this paper can be used to decide on the order of Padé approximations, with many other linear controllers including robust controllers with regards to model uncertainties.

The outline of this paper is as follows. Section II introduces a model of CACC-controlled vehicle string with both the vehicle actuator delay and the wireless communication delay. Section III presents a method to pursue the appropriate order of the Padé approximation in view of string stability, in the frequency-domain. In Section IV, the proposed Padé approximation order is validated by means of time-domain simulations. Section V summarizes the conclusions.

\section{CACC WITH DELAYS}

\section{A. Delays in a CACC String}

A homogeneous CACC string is considered in this paper, as shown in Fig. 1, where $l, q_{i}, v_{i}$, and $u_{i}$ are the length, position, velocity, and desired acceleration of vehicle $i$, respectively; $d_{r, i}$ represents the desired distance between vehicle $i$ and its pre-

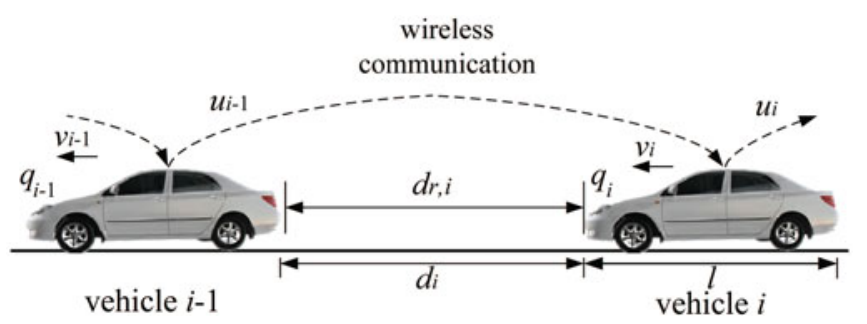

Fig. 1. CACC-equipped string of vehicles.

ceding vehicle $i-1$, and $d_{i}$ is the actual intervehicle distance. Focussing on the analysis of delays in a CACC system, a onevehicle look-ahead topology is adopted. It has been shown in [20] that a one-vehicle look-ahead topology leads to smaller admissible time gaps than a multiple-vehicle look-ahead topology below a certain break-even communication delay. In a homogeneous vehicle string, the wireless communication delay $\theta_{c}$ and the vehicle length $l$ are independent of the vehicle index $i$.

Due to nonlinear dynamics of engine and drive train, aerodynamic drag, and rolling resistance, feedback linearization is often adopted to arrive at a simplified model for CACC design [21]. However, time delays cannot be omitted. Adopting [7], the acceleration $a_{i}$ of vehicle $i$ is as follows:

$$
\tau \dot{a}_{i}(t)+a_{i}=u_{i}\left(t-\theta_{a}\right)
$$

where $\theta_{a}$ represents the vehicle actuator delay, and $\tau$ is a time constant, representing the longitudinal vehicle response. Both $\tau$ and $\theta_{a}$ are set identical for both acceleration and brake situations, for the sake of simplicity. In addition, considering a model gain $k_{g}$ with a nominal value of 1 , the Laplace transfer function from the desired acceleration $U_{i}$ to the position $Q_{i}$ reads

$$
\frac{Q_{i}(s)}{U_{i}(s)}=D_{a}(s) G(s)=e^{-\theta_{a} s} \frac{k_{g}}{s^{2}(\tau s+1)}
$$

where $s \in \mathbb{C}$ is the Laplace variable. Here, the representations of vehicle dynamics is as follows:

$$
\begin{aligned}
D_{a}(s) & =e^{-\theta_{a} s} \\
G(s) & =\frac{k_{g}}{s^{2}(\tau s+1)}
\end{aligned}
$$

are adopted to make it clear when replacing the exact delay by its Padé approximations. This model can adequately describe the longitudinal vehicle dynamics in view of CACC systems [8], [11], [20]. Note that, both $\tau$ and $k_{g}$ are uncertain in practice. Thus, a variety of vehicle parameters will be considered in the next of this paper to pursue a suitable order of Padé approximations.

In a CACC system, the wireless intervehicle link is employed for feedforward purposes. In our case, the feedforward input is the desired acceleration of the preceding vehicle, i.e., $u_{i-1}$ (see Section II-B). Although the wireless communication time delay has decreased largely with the recent developments of both software and hardware [12], [20], it still has a significant effect on string stability of CACC systems. Thus, considering the wireless communication $\theta_{c}$, the actual feedforward input of 
vehicle $i$ is

$$
u_{i-1, c}(t)=u_{i-1}\left(t-\theta_{c}\right)
$$

where $\theta_{c}$ consists of the total time delay due to queueing, contention, transmission, and propagation. Note that other network effects in wireless communication, such as packet loss and sampling intervals, are not taken into account in this paper.

\section{B. CACC Controller}

In a CACC string, each vehicle (except the leading one) aims to keep a desired distance $d_{r, i}$ to its preceding vehicle. The constant time gap policy is utilized, which is the most common one to improve string stability, see [2] and the references contained therein. Consequently, the desired intervehicle distance involves a standstill distance and a velocity-dependent part:

$$
d_{r, i}(t)=r+h v_{i}(t)
$$

where $h$ is a constant time gap, and $r$ is the standstill distance, which are identical for all vehicles in the string. Pursuing a small value of $h$ can increase traffic throughput. However, inherent time delays in the wireless communication yield a lower bound for the time gap from a string-stability perspective [2], [7].

The actual intervehicle distance $d_{i}$ reads

$$
d_{i}(t)=q_{i-1}(t)-q_{i}(t)-l
$$

which is measured by the radar sensor. To realize the vehicle-following objective, the intervehicle distance error $e_{i}$, defined as

$$
e_{i}(t)=d_{i}(t)-d_{r, i}(t)
$$

should asymptotically converge to zero. To this end, a variety of controllers have been proposed [4], [8], [9], [13], [16], [20], [22]. However, a linear PD controller is most widely adopted, especially in experimental applications [7], [14]. Here, we adopted the algorithm in [7], where the Routh-Hurwitz stability criterion is applied to analyze the stability of PD controlled CACC systems with no vehicle actuator delay. The control structure is briefly introduced as follows:

with $k_{g}=1$, the third derivative of (7) reads

$$
\dddot{e}_{i}(t)=-\frac{1}{\tau} \ddot{e}_{i}(t)-\frac{1}{\tau} \xi_{i}(t)+\frac{1}{\tau} u_{i-1}\left(t-\theta_{c}\right)
$$

where a new input $\xi_{i}$ is defined as

$$
\xi_{i}(t):=h \dot{u}_{i}(t)+u_{i}(t) .
$$

To stabilize the error dynamics, the control law for $\xi_{i}$ follows:

$$
\xi_{i}(t)=u_{i-1}\left(t-\theta_{c}\right)+\omega_{p} e_{i}(t)+\omega_{d} \dot{e}_{i}(t) .
$$

In the Laplace domain, a standard PD controller reads

$$
K(s)=\omega_{p}+\omega_{d} s
$$

where $\omega_{p}$ and $\omega_{d}$ represent the proportional and derivative parameters, respectively. $\omega_{p}$ is designed to be $\omega_{d}^{2}$ for notational and analytic convenience, (see also [2], [19]), which can reduce an overflow of controller parameters in the sequel.

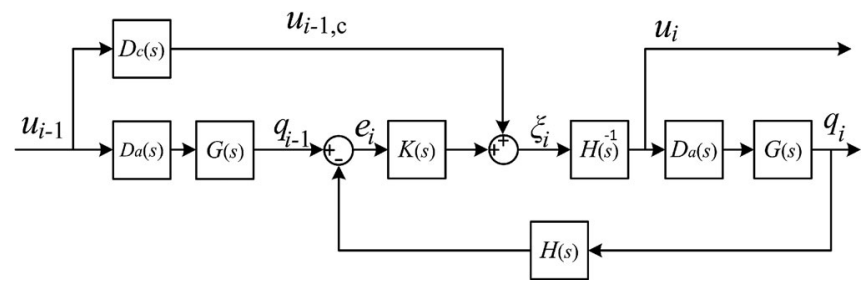

Fig. 2. Block scheme of the CACC system.

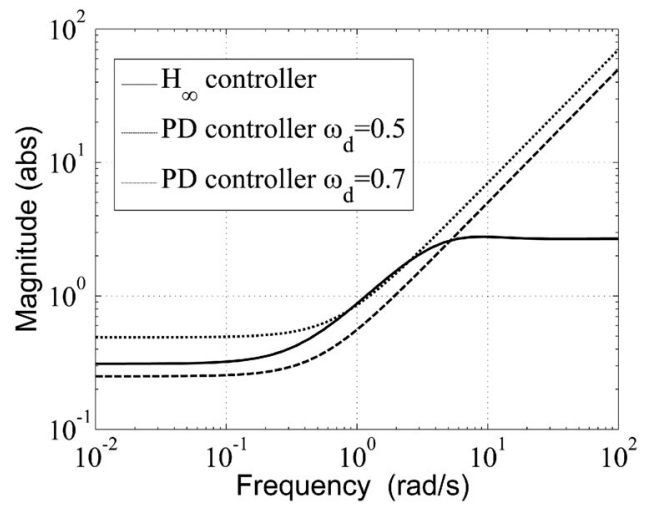

Fig. 3. Comparison of PD and $\mathcal{H}_{\infty}$ controllers.

Consequently, the control structure with both wireless communication delay $\theta_{c}$ and vehicle actuator delay $\theta_{a}$ can be depicted as in Fig. 2, where $H(s)$ and $D_{c}(s)$ represent the Laplace transforms

$$
\begin{gathered}
H(s)=h s+1 \\
D_{c}(s)=e^{-\theta_{c} s} .
\end{gathered}
$$

\section{Comparison of $P D$ and $\mathcal{H}_{\infty}$ Controllers}

In order to determine a suitable approximation order of time delays in CACC systems, we need to consider a variety of vehicle parameters and communication delays. Although controller design is not pursued in this paper, PD controllers can perform similar to other linear controllers in the frequency-range of interest for this particular application, such as an $\mathcal{H}_{\infty}$ controller [20]. To motivate this statement, Fig. 3 shows the magnitudes of the $\mathcal{H}_{\infty}$ feedback controller in [20] and the PD controller (11) with $\omega_{d}=0.5$ and 0.7 . At frequencies higher than $10 \mathrm{rad} / \mathrm{s}$, PD controllers cannot eliminate the signal noise as the $\mathcal{H}_{\infty}$ controller, which has a higher order. However, the similarity in both frequency responses indicates that the method to choose a suitable order of Padé approximations with one particular PD controller can be used for other linear controllers, although the resulting approximation order may be different.

\section{CACC Stability Based on PadÉ Approximation}

In this section, we examine the suitable order of the Padé approximations of both the actuator and the communication delays in view of individual vehicle stability and string stability. 


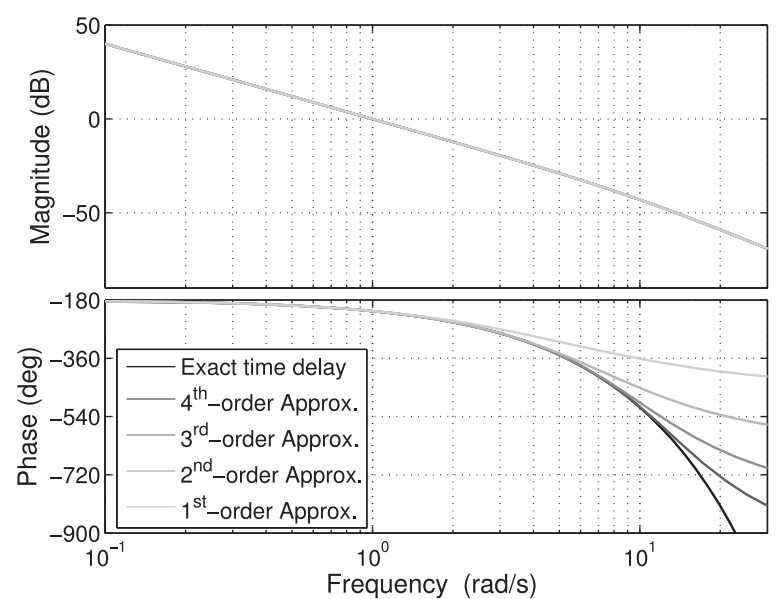

Fig. 4. Frequency-domain responses of vehicle dynamics with exact and approximated delays.

\section{A. Padé Approximation}

Padé approximations of a delay yield a finite-dimensional model with a rational transfer function, of which the expressions with the same order of the numerator and the denominator are as follows:

$$
e^{-T s} \cong \frac{\sum_{k=0}^{p} \beta_{k}(-T s)^{k}}{\sum_{k=0}^{p} \beta_{k}(T s)^{k}}
$$

where $T \in\left\{\theta_{a}, \theta_{c}\right\}$ represents the time delay, and $p$ is the order of the Padé approximation. The coefficients $\beta_{k}$ are given by

$$
\beta_{k}=\frac{(2 p-k) ! p !}{(2 p) ! k !(p-k) !}
$$

Increasing the order can lead to a better approximation, while the resulting model is more complex. The 1st-, 2nd-, 3rd-, and 4th-order Padé approximations of $e^{-T s}$ are represented as

$$
\begin{aligned}
& P_{1}(s)=\frac{1-\frac{1}{2} T s}{1+\frac{1}{2} T s} \\
& P_{2}(s)=\frac{1-\frac{1}{2} T s+\frac{1}{12}(T s)^{2}}{1+\frac{1}{2} T s+\frac{1}{12}(T s)^{2}} \\
& P_{3}(s)=\frac{1-\frac{1}{2} T s+\frac{1}{10}(T s)^{2}-\frac{1}{120}(T s)^{3}}{1+\frac{1}{2} T s+\frac{1}{10}(T s)^{2}+\frac{1}{120}(T s)^{3}} \\
& P_{4}(s)=\frac{1-\frac{1}{2} T s+\frac{3}{28}(T s)^{2}-\frac{1}{84}(T s)^{3}+\frac{1}{1680}(T s)^{4}}{1+\frac{1}{2} T s+\frac{3}{28}(T s)^{2}+\frac{1}{84}(T s)^{3}+\frac{1}{1680}(T s)^{4}} .
\end{aligned}
$$

Then, the frequency-domain responses for the exact and approximated transfer functions from the desired acceleration $u_{i}$ to the position $q_{i}$, i.e., $D_{a}(s) G(s)$ are shown in Fig. 4, with $\tau=0.1 \mathrm{~s}$ and $\theta_{a}=0.5 \mathrm{~s}$ as an example. In the frequencydomain responses, the magnitudes of exact and approximated models have hardly visible differences, which are smaller than $0.02 \mathrm{~dB}$, while the phases differ for the higher frequencies. Since choosing a suitable order of the Padé approximation mainly depends on the system dynamics, it is necessary to apply a large range of parameters, to find an approximation order leading to the sufficiently similar stability properties as the aforementioned CACC system with exact delays.

Next, we will check the individual-vehicle-stable PD controller parameter regions and the minimum string-stable time gaps with the exact and approximated models, respectively.

\section{B. Individual Vehicle Stability}

Individual vehicle stability, as mentioned in the previous section, requires that the system should have a stable equilibrium. With the selected order Padé approximation of the delays, the resulting CACC system and the one with exact delays should have approximately the same (individual) stability properties. Thus, the PD controller parameter region for which the approximated system is stable, needs to be close enough to the stable PD controller parameter region for the system with the exact time delay. We start with a 2nd-order Padé approximation, due to the inaccuracy of the 1st-order Padé approximation.

Considering the control structure shown in Fig. 2, the transfer function from the desired acceleration to the intervehicle distance error reads

$$
C(s)=\frac{D_{a}(s) G(s)\left(1-D_{c}(s)\right)}{1+D_{a}(s) G(s) K(s)} .
$$

It is clear that the communication delay $\theta_{c}$ occurring in the feedforward loop does not influence individual vehicle stability in the presented CACC structure.

First, we consider the stable PD controller region in case of the CACC model with exact delays. Making use of the Nyquist stability criterion, the PD controller parameter region to guarantee individual vehicle stability can be graphically determined. In Fig. 2, the open loop transfer function reads

$$
L(s)=D_{a}(s) G(s) K(s)
$$

which should not clock-wise encircle the point $(-1,0)$ since the system is stable.

Next, considering the CACC system with the Padé approximated time delays, the Routh-Hurwitz stability criterion can be applied to calculate the stable PD controller region. In case of ideal vehicle dynamics, where no vehicle actuator delay exists, i.e., $\theta_{a}=0$, it follows that the CACC string can be stabilized for $0<\omega_{d}<\frac{1}{\tau}$ [23]. Now, consider $\theta_{a}>0$. Substituting (3), (11), and (13) in (16), we obtain finite-dimensional transfer functions, such that the Routh-Hurwitz stability criterion can be applied. The roots of the denominator of (16), i.e.,

$$
\begin{aligned}
& 1+D_{a}(s) G(s) K(s) \\
& =1+\frac{k_{g}\left(\omega_{p}+\omega_{d} s\right) \sum_{k=0}^{p} \beta_{k}\left(-\theta_{a} s\right)^{k}}{s^{2}(\tau s+1) \sum_{k=0}^{p} \beta_{k}\left(\theta_{a} s\right)^{k}}
\end{aligned}
$$

should be in the left half of the complex plane to achieve stable systems. Thus, it requires a positive $\omega_{d}$ limited to a maximum value $\omega_{d, \max }$ to guarantee individual vehicle stability, i.e., leading to the stable PD controller parameter region

$$
\omega_{d} \in\left(0, \omega_{d, \max }\left(\theta_{a}, \tau\right)\right) .
$$


TABLE I

Stable PD Controller Gains $\omega_{d, \text { max }}\left(\theta_{a}, \tau\right)$ Without the Vehicle ACTUATOR DELAY; AND WITH THE VEHICLE ACTUATOR DELAY, AND ITS 2ND-, 3RD-, AND 4TH-ORDER PADÉ APPROXIMATIONS

\begin{tabular}{lccc}
\hline \hline & \multicolumn{3}{c}{$\omega_{d, \max }\left(\theta_{a}, \tau\right)$} \\
\cline { 2 - 4 } case & $\tau=0.1$ & $\tau=0.3$ & $\tau=0.5$ \\
\hline$\theta_{a}=0$ & 10 & 3.333333 & 2 \\
exact delay $\theta_{a}=0.1$ & 3.7732 & 2.0830 & 1.4577 \\
2nd-order Padé of $\theta_{a}=0.1$ & 3.776279 & 2.083767 & 1.458203 \\
3rd-order Padé of $\theta_{a}=0.1$ & 3.771906 & 2.083407 & 1.458121 \\
4th-order Padé of $\theta_{a}=0.1$ & 3.776158 & 2.083763 & 1.458203 \\
exact delay $\theta_{a}=0.3$ & 1.7980 & 1.2577 & 0.9840 \\
2nd-order Padé of $\theta_{a}=0.3$ & 1.800136 & 1.258760 & 0.984279 \\
3rd-order Padé of $\theta_{a}=0.3$ & 1.793552 & 1.257302 & 0.983764 \\
4th-order Padé of $\theta_{a}=0.3$ & 1.799742 & 1.258719 & 0.984271 \\
exact delay $\theta_{a}=0.5$ & 1.1909 & 0.9157 & 0.7546 \\
2nd-order Padé of $\theta_{a}=0.5$ & 1.191522 & 0.916885 & 0.755256 \\
3rd-order Padé of $\theta_{a}=0.5$ & 1.185621 & 0.914896 & 0.754381 \\
4th-order Padé of $\theta_{a}=0.5$ & 1.191091 & 0.916803 & 0.755232 \\
\hline \hline
\end{tabular}

This stability region thus includes the case of $\theta_{a}=0$, with $\omega_{d, \max }(0, \tau)=\frac{1}{\tau}$. Applying 2nd-, 3rd-, and 4th-order Padé approximations of $e^{-\theta_{a} s}$, respectively, the corresponding values of $\omega_{d, \max }\left(\theta_{a}, \tau\right)$ can be obtained.

As a result, Table I shows the maximum PD controller gain $\omega_{d, \max }\left(\theta_{a}, \tau\right)$ in view of individual vehicle stability with parameters $\theta_{a} \in\{0.1,0.3,0.5\} \mathrm{s}$ and $\tau \in\{0.1,0.3,0.5\} \mathrm{s}$. Here, the model gain is assumed with the nominal value $k_{g}=1$. With different $k_{g}=\bar{k}_{g}$, the values of $\omega_{d, \max }\left(\theta_{a}, \tau\right)$ with exact and approximated delays change with the same proportion, which has no effect on the accuracy of Padé approximations. Note that adopting the Padé approximations does not guarantee that the resulting $\omega_{d, \max }\left(\theta_{a}, \tau\right)$ is smaller than the one obtained from the Nyquist mapping of the model with exact delays. Table I indicates that the values of $\omega_{d, \max }\left(\theta_{a}, \tau\right)$ for CACC systems with 2nd- and 4th-order Padé approximations are slightly larger than these for the system with the exact delay, whereas representing the CACC system with 3rd-order Padé approximations results in smaller values of $\omega_{d, \max }\left(\theta_{a}, \tau\right)$ except the cases of $\theta_{a}=0.1 \mathrm{~s}$ and $\tau \in\{0.3,0.5\} \mathrm{s}$. However, in spite of the inaccuracy of graphical Nqyuist methods and the calculations from the Routh-Hurwitz criterion, the values of $\omega_{d \text {, max }}\left(\theta_{a}, \tau\right)$ for 2nd-, 3rd-, and 4th-order approximated CACC systems are all sufficiently close to the one for the original system. Since a smaller value of $\omega_{d, \max }\left(\theta_{a}, \tau\right)$ will never compromise stability of the original system, it is reasonable to apply the 3rd-order Padé approximation on the vehicle actuator delay in a CACC system.

In the next section, we check the suitable order Padé approximation from a string stability perspective.

\section{String Stability}

Since the constant time gap policy is introduced to achieve string stability, we choose the minimum string-stable time gap as the string stability property to arrive at the lowest possible Padé approximation order. In other words, the minimum stringstable time gaps for the CACC system with approximated delays and with exact delays should be sufficiently close. To this end, a range of values for the parameters of the vehicle actuator delays, the communication delays, and the PD controller gain are considered. Because of the results of the previous section, we start with the 3rd-order Padé approximation to find the suitable order in view of string stability.

In the widely applied performance-oriented approach, string stability is characterized by the amplification in upstream direction of the signal of interest [2], [7]. Define the string stability transfer function as $S(s)$, which describes the relation between a relevant (scalar) signal of vehicle $i$ and the corresponding signal of its preceding vehicle $i-1$. In CACC systems, the signals of interest generally involve the intervehicle distance error, the acceleration, the velocity, and the position. Then the system of CACC-controlled vehicles is string stable if

$$
\sup _{\omega}|S(j \omega)| \leq 1
$$

with the frequency $\omega \in \mathbb{R}^{+}$.

Let $E_{i}(s), U_{i}(s), V_{i}(s)$, and $Q_{i}(s)$ represent the Laplace transforms of the intervehicle distance error, the vehicle input (desired acceleration), the velocity, and the position of vehicle $i$, respectively. The velocity is chosen in view of traffic analysis [7]. The position has to be considered for heterogeneous traffic [2], whereas the acceleration is concerned for vehicle throttle/brake constraints, and the intervehicle error presents the vehicle following accuracy. Under the assumption of homogeneous traffic, the string stability transfer function $S(s)$ does not depend on the choice of the signal, yielding

$$
\begin{aligned}
S(s) & =\frac{E_{i}(s)}{E_{i-1}(s)}=\frac{U_{i}(s)}{U_{i-1}(s)}=\frac{V_{i}(s)}{V_{i-1}(s)}=\frac{Q_{i}(s)}{Q_{i-1}(s)} \\
& =\frac{1}{H(s)} \frac{D_{c}(s)+D_{a}(s) G(s) K(s)}{1+D_{a}(s) G(s) K(s)} .
\end{aligned}
$$

Substituting (2), (11), (12), and (21) in (20) yields

$$
\begin{aligned}
& \left|\frac{1}{h j \omega+1}\right| \cdot \\
& \left|\frac{e^{-\theta_{c} j \omega}+\frac{k_{g}}{(j \omega)^{2}(\tau j \omega+1)} e^{-\theta_{a} j \omega}\left(\omega_{p}+\omega_{d} j \omega\right)}{1+\frac{k_{g}}{(j \omega)^{2}(\tau j \omega+1)} e^{-\theta_{a} j \omega}\left(\omega_{p}+\omega_{d} j \omega\right)}\right| \leq 1
\end{aligned}
$$

to realize string stability.

In the case without communication delay, (20) reduces to

$$
\sup _{\omega}\left|\frac{1}{h j \omega+1}\right| \leq 1
$$

which is fulfilled for any nonnegative time gap, i.e., $h \geq 0 \mathrm{~s}$. However, as previously stated, in reality a communication delay exists, which plays a significant role in designing the time gap for string stability.

When $\theta_{c}>0 \mathrm{~s}$, the magnitude of the string stability transfer function can be expressed as

$$
|S(j \omega)|=\frac{1}{\sqrt{(h \omega)^{2}+1}}\left|\frac{M(j \omega)}{N(j \omega)}\right|
$$




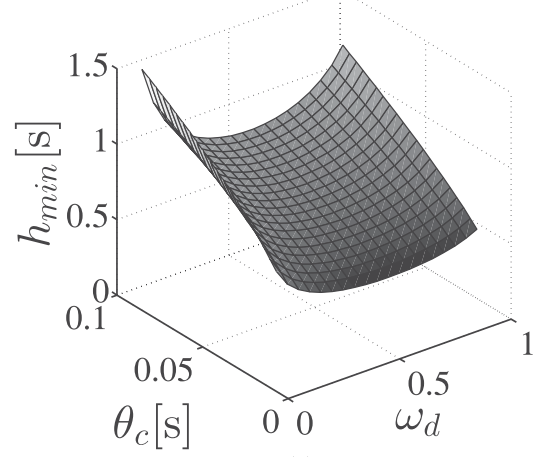

(a)

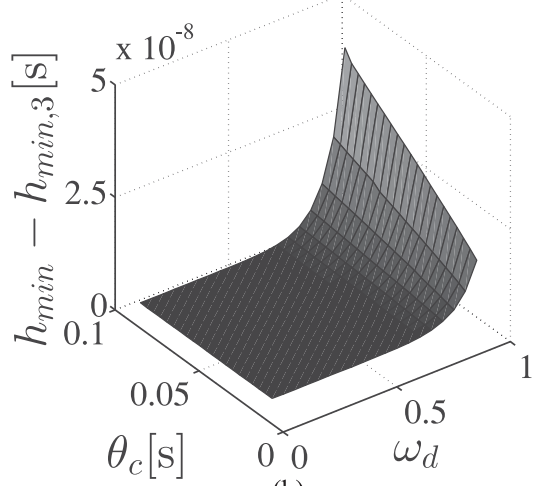

(b)

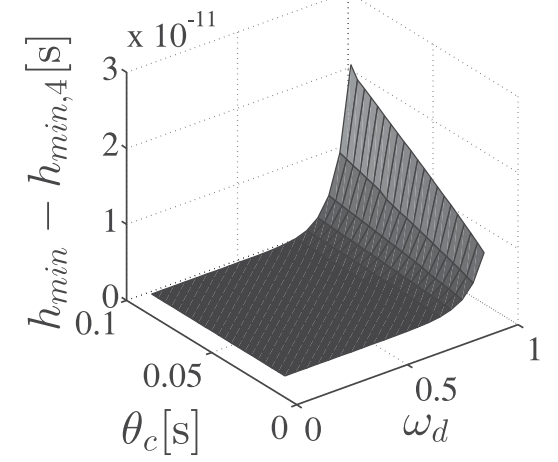

(c)

Fig. 5. (a) Minimum string-stable time gap $h_{\mathrm{m} \text { in }}$ for CACC with exact delays; difference $h_{\mathrm{m} \text { in }}-h_{\mathrm{min}, p}$ of (b) the 3rd-order Padé approximation and of (c) the 4 th-order Padé approximation, with $\tau=0.3 \mathrm{~s}, \theta_{a}=0.3 \mathrm{~s}, \theta_{c} \in[0.02,0.1] \mathrm{s}, k_{g}=1$, and $\omega_{d} \in[0.1,1.0]$.

where

$$
\begin{aligned}
M(j \omega) & =e^{-\theta_{c} j \omega} \\
& +\frac{k_{g}}{(j \omega)^{2}(\tau j \omega+1)} e^{-\theta_{a} j \omega}\left(\omega_{p}+\omega_{d} j \omega\right) \\
N(j \omega) & =1+\frac{k_{g}}{(j \omega)^{2}(\tau j \omega+1)} e^{-\theta_{a} j \omega}\left(\omega_{p}+\omega_{d} j \omega\right)
\end{aligned}
$$

from which it follows that string stability can be guaranteed for $h \geq h_{\min }$, where

$$
h_{\min }=\sup _{\omega}\left(\frac{\sqrt{\left|\frac{M(j \omega)}{N(j \omega)}\right|^{2}-1}}{\omega}\right) .
$$

Substituting (15) and (25) in (26), results in an approximated minimum time gap $h_{\min , p}$, where $p$ represents the order of Padé approximation.

To judge the quality of Padé approximations for the vehicle actuator delay and the communication delay, we adopt the minimum string-stable time gap as the criterion in the sense that the system with approximated delays should yield a minimum time gap $h_{\min , p}$ which is close to $h_{\min }$. Note that $h_{\min , p}$ is not guaranteed to be larger than $h_{\min }$. The values of vehicle actuator lag $\tau \in[0.1,0.5] \mathrm{s}$ and model gain $k_{g} \in[0.5,1.5] \mathrm{s}$ are adopted, so that different vehicle dynamics are considered. It is necessary to compare the values of $h_{\min }$ and $h_{\min , p}$ with a range of vehicle actuator delays and communication delays. Thus, $\theta_{a} \in[0.1,0.5] \mathrm{s}$ is considered. In view of the communication delay, the vehicle motion data update frequency varies from $10 \mathrm{~Hz}$ in the GCDC 2011 [8]-[11] to $25 \mathrm{~Hz}$ in [20], with the development of both hardware and software. In the GCDC 2016 [12], the same update rate as in [20] was used. Thus, the communication delay indeed is $\theta_{c} \cong 0.02 \mathrm{~s}$. In this paper, we took the range $\theta_{c} \in[0.02,0.1] \mathrm{s}$. The upper bound $\theta_{c}=0.1 \mathrm{~s}$, also mentioned as a communication requirement in [12], which tolerates more calculation time due to complex controllers or more information communicated.

First, with a constant vehicle actuator delay $\theta_{a}=0.3 \mathrm{~s}$, we choose the vehicle parameters $\tau=0.3 \mathrm{~s}$ and $k_{g}=1$, and a range of communication delay $\theta_{c} \in[0.02,0.1] \mathrm{s}$, in order to judge the quality of Padé approximations with different PD controller parameters. Table I indicates that $\omega_{d, \max }\left(\theta_{a}, \tau\right)=1.2577$ for the system with an exact time delay. Thus, selecting $\omega_{d} \in[0.1,1.0]$ for the sake of individual vehicle stability, Fig. 5 shows the minimum time gap $h_{\min }$ with the nominal value of $1 \mathrm{~s}$, and the minimum string-stable time gap difference $h_{\min }-h_{\min , p}$. Time gaps above the surface in Fig. 5(a) will guarantee string stability of the CACC string. Fig. 5(b) and (c) indicate that the difference $h_{\min }-h_{\min , p}$ is less than $5.0 \times 10^{-8} \mathrm{~s}$ and $3.0 \times 10^{-11} \mathrm{~s}$ for 3rd- and 4th-order Padé approximations, respectively, which are sufficiently small. Hence, there is no necessity to consider a higher order Padé approximation.

Then, choosing the maximum communication delay $\theta_{c}=$ $0.1 \mathrm{~s}$, we study how the vehicle parameters $\tau$ and $k_{g}$ influence the accuracy of the Padé approximations. The PD controller parameter $\omega_{d}=0.6$ is selected, considering the controller comparison in Section II-C and the individual vehicle stability requirement in Table I. Fig. 6 shows $h_{\min }$ and $h_{\min }-h_{\min , p}$ as a function of $\tau \in[0.1,0.5] \mathrm{s}$ and $\theta_{a} \in[0.1,0.5] \mathrm{s}$, while $k_{g}=1$ is adopted. With $k_{g} \in[0.5,1.5]$ and $\tau=0.3 \mathrm{~s}, h_{\min }$ and $h_{\min }-h_{\min , p}$ are presented in Fig. 7. Both Figs. 6 and 7 indicate that vehicle parameters indeed affect the quality of Padé approximations, i.e., $h_{\min }-h_{\min , p}$ increases with increasing $k_{g}$ and $\tau$. This may seem counter-intuitive, but realize that, although $h_{\min }-h_{\min , p}$ would increase with smaller $\tau$ when only accounting for the same frequency, different values of $\tau$ lead to the corresponding $h_{\min }$ at different frequencies. Thus, the frequency also influences the values of $h_{\min }-h_{\min , p}$.

Finally, both time delays are considered with a range of values, i.e., $\theta_{c} \in[0.02,0.1] \mathrm{s}$ and $\theta_{a} \in[0.1,0.5] \mathrm{s}$. According to Figs. 6 and $7, k_{g}=1.5$ and $\tau=0.5 \mathrm{~s}$ are adopted to check the difference $h_{\min }-h_{\min , p} . \omega_{d}=0.6$ can still keep individual vehicle stability $\left(\omega_{d, \max }\left(\theta_{a}, \tau\right) \cong 0.6596\right.$, with $\theta_{a}=0.5 \mathrm{~s}$, $\tau=0.1 \mathrm{~s}$ and $k_{g}=1.5$ ), while the stable margin gets smaller than that in Table I with $k_{g}=1$. The numerical results are shown in Fig. 8. In Fig. 8(a), with exact delays, the minimum time gap $h_{\mathrm{min}}$ increases with both the vehicle actuator delay and the wireless communication delay. In Fig. 8(b) and (c), the differences of the minimum time gap for the 3rd- and 4th-order Padé approximated delays are less than $1.0 \times 10^{-6} \mathrm{~s}$ and $1.0 \times 10^{-9} \mathrm{~s}$, 


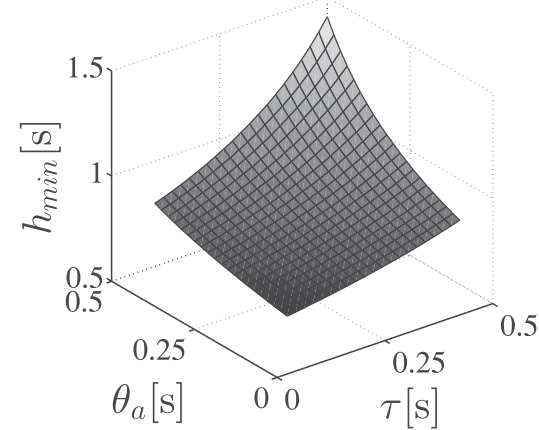

(a)

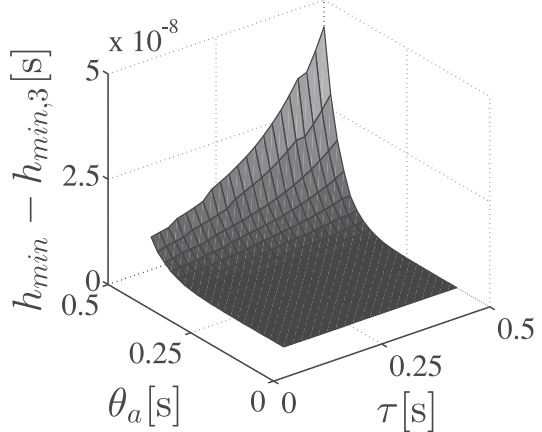

(b)

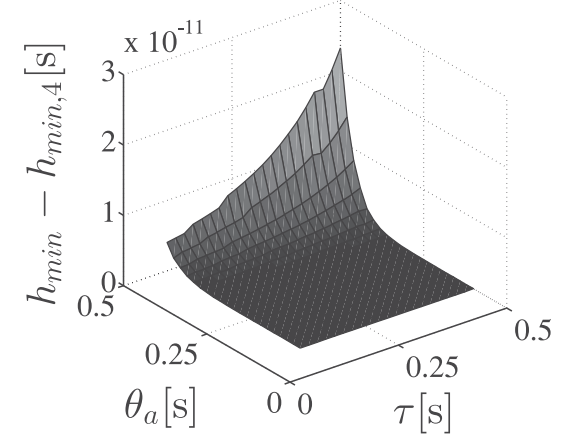

(c)

Fig. 6. (a) Minimum string-stable time gap $h_{\mathrm{m} \text { in }}$ for CACC with exact delays; difference $h_{\mathrm{min}}-h_{\min , p}$ of (b) the 3rd-order Padé approximation and of (c) the 4th-order Padé approximation, with $\tau \in[0.1,0.5] \mathrm{s}, \theta_{a} \in[0.1,0.5] \mathrm{s}, \theta_{c}=0.1 \mathrm{~s}, k_{g}=1$, and $\omega_{d}=0.6$.

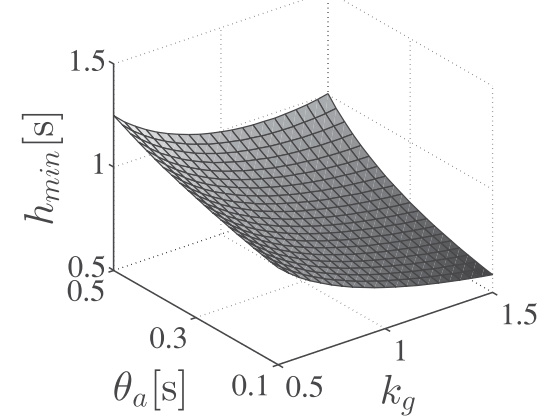

(a)

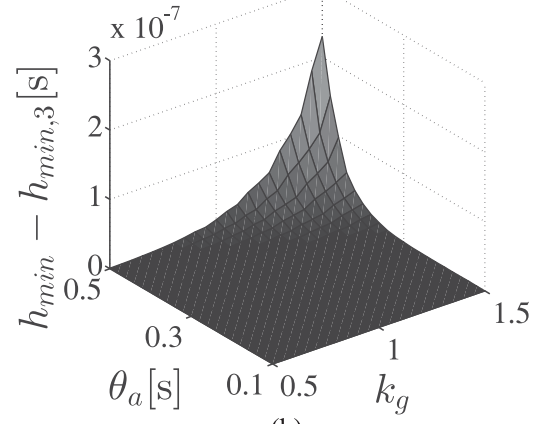

(b)

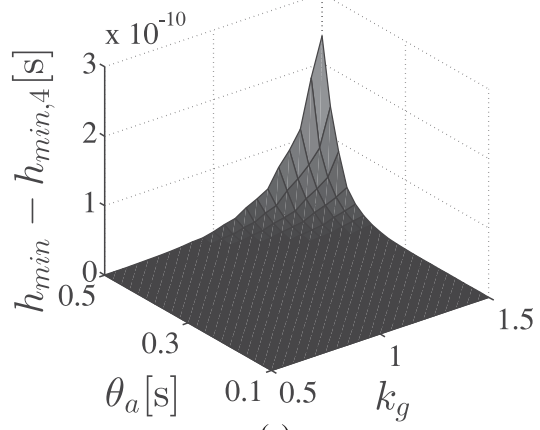

(c)

Fig. 7. (a) Minimum string-stable time gap $h_{\mathrm{m} \text { in }}$ for CACC with exact delays; difference $h_{\mathrm{m} \text { in }}-h_{\min , p}$ of (b) the 3rd-order Padé approximation and of (c) the 4th-order Padé approximation, with $\tau=0.3 \mathrm{~s}, k_{g} \in[0.5,1.5], \theta_{a} \in[0.1,0.5] \mathrm{s}, \theta_{c}=0.1 \mathrm{~s}$, and $\omega_{d}=0.6$.

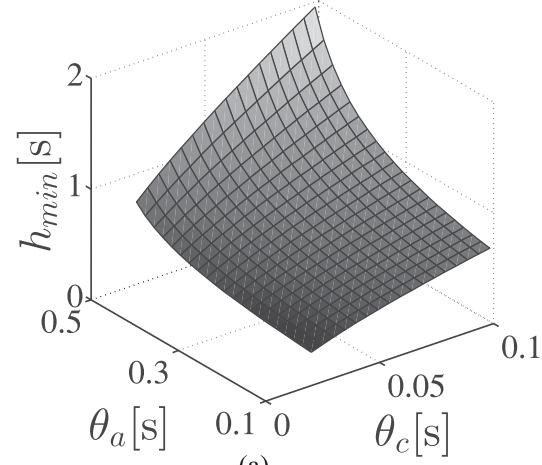

(a)

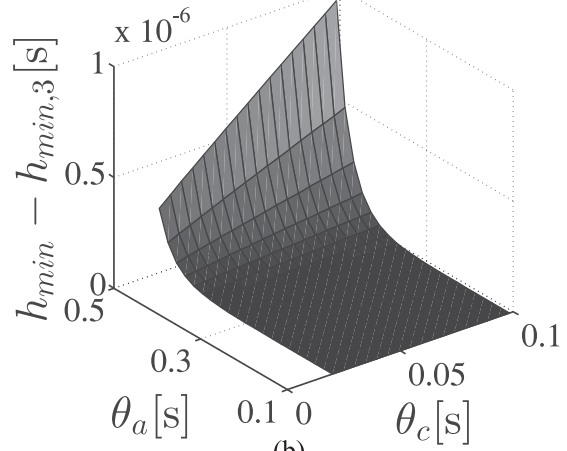

(b)

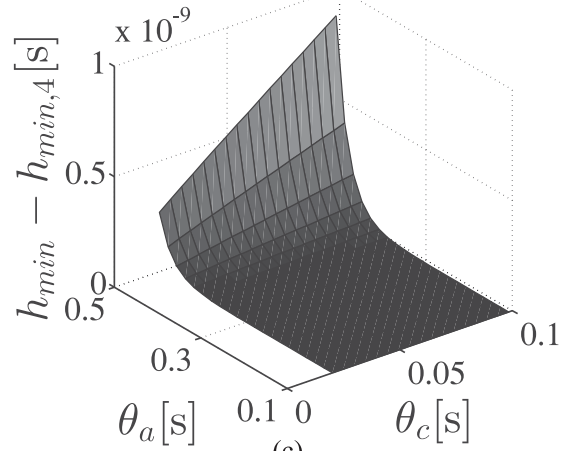

(c)

Fig. 8. (a) Minimum string-stable time gap $h_{\mathrm{m} \text { in }}$ for CACC with exact delays; difference $h_{\mathrm{m} \text { in }}-h_{\min , p}$ of (b) the 3rd-order Padé approximation and of (c) the 4th-order Padé approximation, with $\tau=0.5 \mathrm{~s}, k_{g}=1.5, \theta_{a} \in[0.1,0.5] \mathrm{s}, \theta_{c} \in[0.02,0.1] \mathrm{s}$, and $\omega_{d}=0.6$.

respectively. Note that different PD controller gains can be used to pursue the driving comfort or to decrease the intervehicle distance error, whereas the resulting difference $h_{\min }-h_{\min , p}$ has the similar accuracy level. Therefore, adopting the 3rd- and 4th-order Padé approximations in this CACC setting is sufficient in view of string stability.

If independent proportional and derivative parameters are considered, numerical analyses of the quality of Padé approximations involve one more parameter, and rather complex simulation study is required. However, the procedure proposed in this paper can also be followed.
In the given setting of the CACC system, taking a 3rd-order Padé approximation for both the vehicle actuator delays and the communication delays is suitable from the perspective of string stability properties.

\section{Simulation In Time-Domain}

To validate the accuracy of the selected order Padé approximation by the proposed method, time-domain simulations have been carried out for a CACC string with four vehicles. In this particular case, the 3rd-order Padé approximation is chosen 


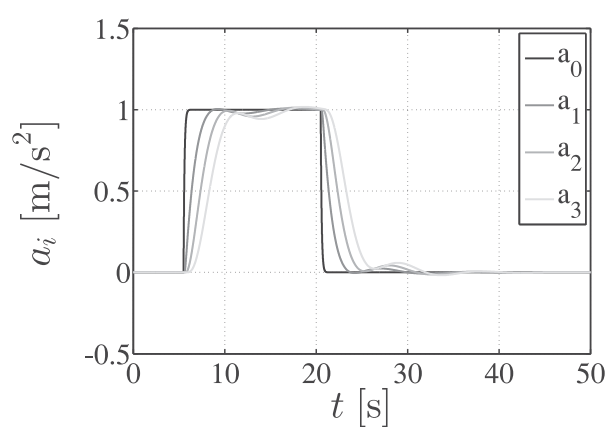

(a)

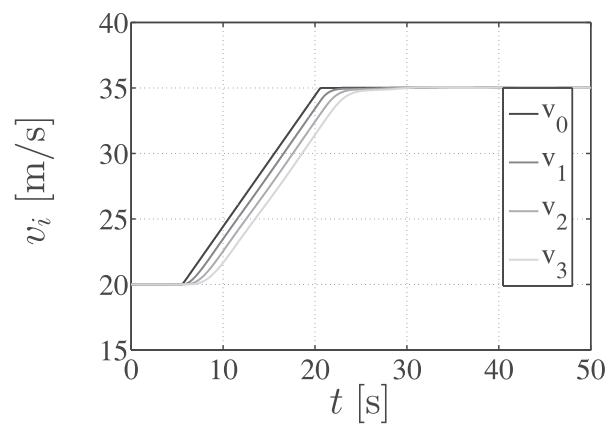

(b)

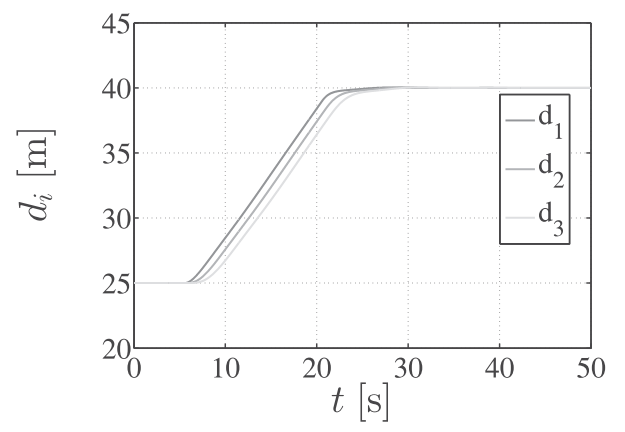

(c)

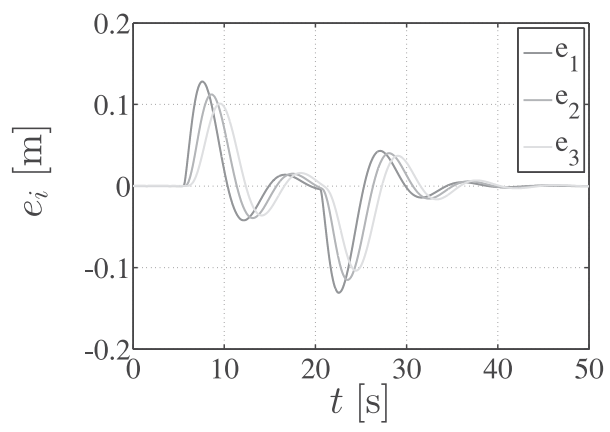

(d)

Fig. 9. Time responses of (a) the acceleration $a_{i}$, (b) the velocity $v_{i}$, (c) the intervehicle distance $d_{i}$, and (d) the distance error $e_{i}$ of CACC with exact delays.

according to the simulation results in Section III. The standstill distance is set as $r=5 \mathrm{~m}$ and the length of vehicle as $l=3 \mathrm{~m}$. Since larger delays can show the difference between models with the exact and with approximated delays more clearly, $\theta_{a}=0.5 \mathrm{~s}$ and $\theta_{c}=0.1 \mathrm{~s}$ are chosen. The value of the model gain $k_{g}=1$ is chosen. Considering a smaller $\tau=0.1 \mathrm{~s}$ leads to a faster response of the vehicle model. The PD controller gain is chosen

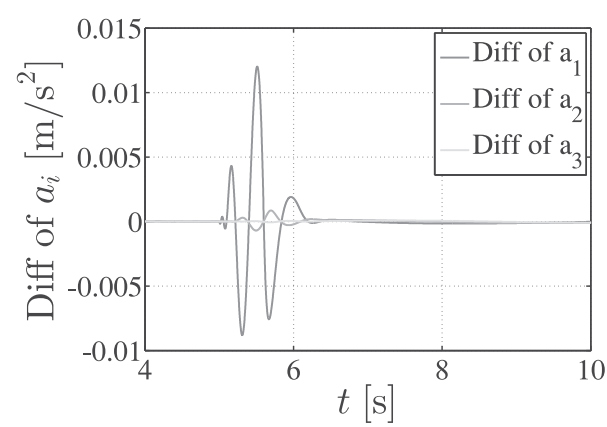

(a)

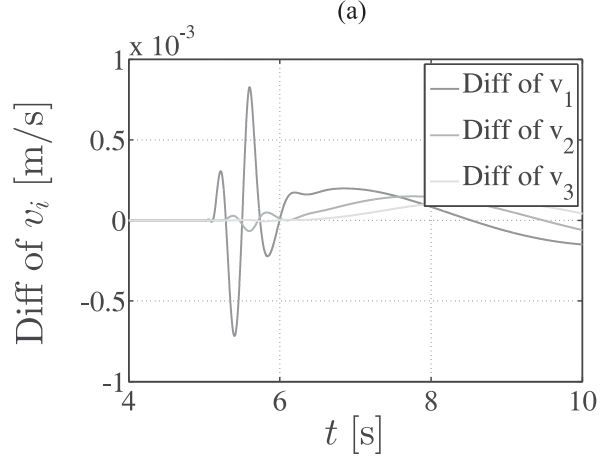

(b)

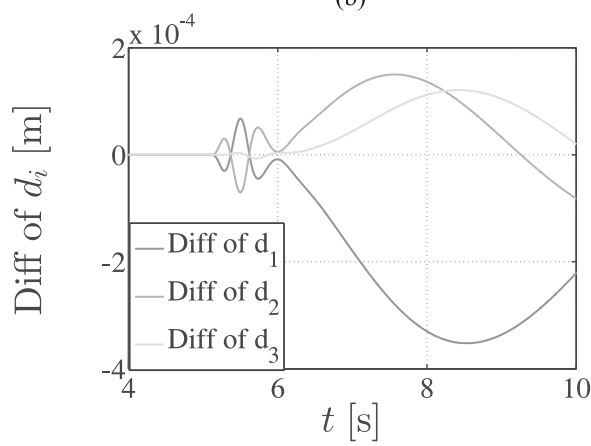

(c)

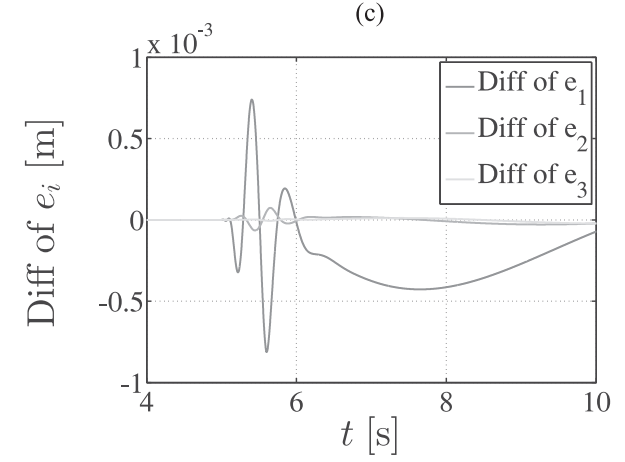

(d)

Fig. 10. Differences of time responses of (a) the acceleration $a_{i}-a_{i, 3}$, (b) the velocity $v_{i}-v_{i, 3}$, (c) the intervehicle distance $d_{i}-d_{i, 3}$, and (d) the distance error $e_{i}-e_{i, 3}$, between the CACC models with exact delays and with 3rd-order Padé approximated delays.

as $\omega_{d}=0.6$ and the time gap as $h=1 \mathrm{~s}$ to guarantee string stability.

In view of string stability, the vehicle acceleration is the most significant disturbance. In addition, the accuracy of approximations of delays is the most sensitive to the step response. Thus, in the time-domain simulations, the desired acceleration for the 
leading vehicle is set as

$$
u_{0}= \begin{cases}1 \mathrm{~m} / \mathrm{s}^{2} & 5 \mathrm{~s} \leqslant t \leqslant 20 \mathrm{~s} \\ 0 & \text { other. }\end{cases}
$$

The initial velocity of all vehicles in this CACC string is $v_{\text {initial }}=20 \mathrm{~m} / \mathrm{s}$. All vehicles start with the desired distance. The acceleration duration is set as $15 \mathrm{~s}$ in order to create enough time for the followers to reach the desired acceleration.

Fig. 9 shows the time responses of the CACC string with exact delays. It is observable that there is a slight overshoot in the acceleration responses in Fig. 9(a). Note that the time gap from the string stability criterion (20) does not guarantee the absence of overshoot in time-domain [7], [24]. The velocity, intervehicle distance, and the distance error responses in Fig. 9(b), (c), and (d), respectively, clearly show that this system is string stable.

Applying the 3rd-order Padé approximation for both the communication delay and the vehicle actuator delay, results in the approximated responses $a_{i, 3}, v_{i, 3}, d_{i, 3}$. The differences between the CACC string responses with exact delays and the ones with approximated delays are shown in Fig. 10, for vehicle $i=1$, 2 , and 3, which are CACC-controlled. We zoom in around $t=5 \mathrm{~s}$, when the acceleration changes and the largest difference occurs. Similar results around $t=20 \mathrm{~s}$ are omitted here. In Fig. 10, all differences decrease with the vehicle number $i$ increasing, due to the sting stable performance. Thus, the responses of the first follower vehicle 1 in the string are considered. In Fig. 10(a), the acceleration differences reach $0.015 \mathrm{~m} / \mathrm{s}^{2}$, which is much smaller than the step input of $1 \mathrm{~m} / \mathrm{s}^{2}$. Accounting for the velocities in Fig. 10(b), the difference due to the 3rdorder Padé approximation of delays is less than $1 \times 10^{-3} \mathrm{~m} / \mathrm{s}$. The differences of intervehicle distances and the distance errors are smaller than $4 \times 10^{-4} \mathrm{~m}$ and $1 \times 10^{-3} \mathrm{~m}$ as shown in Fig. 10(c) and (d), respectively, which are even less than the possible measurement noise of the intervehicle distance.

\section{CONCLUSION}

In a CACC-controlled vehicle string, the communication delay and vehicle actuator delay inherently exist. Adopting a Padé approximation for the delays leads to a rational transfer function representation of the CACC string, which allows many controller design methods. Choosing a low order of Padé approximation, which is accurate enough, can significantly promote the controller design. In this paper, we proposed a method to arrive at a low possible Padé approximation order, based on the individual vehicle stability and the string stability properties for the system with exact delays and with approximated delays. We used a particular PD controller as an example when judging the quality of Padé approximations for a given CACC string. However, the same method can be used in case of other linear controllers. From the individual vehicle stability perspective, we arrived at a suitable order Padé approximation based on the requirement of similar regions for the system with exact delays and the system with approximated delays. The values of the minimum string-stable time gaps for the CACC model were chosen as the string stability criterion to find the suitable approximation order. Different values of vehicle dynamics pa- rameters have been considered. With the results of this method, time-domain simulations were conducted to validate that the selected order Padé approximation was suitable for the given CACC string.

\section{REFERENCES}

[1] G. Marsden, M. McDonald, and M. Brackstone, "Towards an understanding of adaptive cruise control," Transp. Res. C, Emerg. Technol., vol. 9, no. 1, pp. 33-51, 2001

[2] G. J. L. Naus, R. P. A. Vugts, J. Ploeg, M. J. G van de Molengraft, and M. Steinbuch, "String-Stable cacc design and experimental validation: A frequency-domain approach," IEEE Trans. Veh. Technol., vol. 59, no. 9, pp. 4268-4279, Nov. 2010.

[3] S. E. Shladover, D. Su, and X.-Y. Lu, "Impacts of cooperative adaptive cruise control on freeway traffic flow," in Proc. 91 st TRB Annu. Meeting, Jan. 2012, pp. 1-17.

[4] T. Stanger and L. del Re, "A model predictive cooperative adaptive cruise control approach," in Proc. Amer. Control Conf., Jun. 2013, pp. 1374 1379.

[5] W. Heemels and N. van de Wouw, "Stability and stabilization of networked control systems," in Networked Control System. Berlin, Germany: Springer, 2010, pp. 203-253.

[6] X. Liu, A. Goldsmith, S. Mahal, and J. Hedrick, "Effects of communication delay on string stability in vehicle platoons," in Proc. IEEE Intell. Transp. Syst. Conf., Aug. 2001, pp. 625-630.

[7] J. Ploeg, B. Scheepers, E. van Nunen, N. van de Wouw, and H. Nijmeijer, "Design and experimental evaluation of cooperative adaptive cruise control," in Proc. IEEE Intell. Transp. Syst. Conf., Oct. 2011, pp. 260-265.

[8] K. Lidström et al., "A modular CACC system integration and design," IEEE Trans. Intell. Transp. Syst., vol. 13, no. 3, pp. 1050-1061, Sep. 2012.

[9] L. Güvenç et al., "Cooperative adaptive cruise control implementation of team Mekar at the grand cooperative driving challenge," IEEE Trans. Intell. Transp. Syst., vol. 13, no. 3, pp. 1062-1074, Sep. 2012.

[10] A. Geiger et al., "Team AnnieWAY's entry to the 2011 grand cooperative driving challenge," IEEE Trans. Intell. Transp. Syst., vol. 13, no. 3 , pp. 1008-1017, Sep. 2012.

[11] R. Kianfar et al., "Design and experimental validation of a cooperative driving system in the grand cooperative driving challenge," IEEE Trans. Intell. Transp. Syst., vol. 13, no. 3, pp. 994-1007, Sep. 2012.

[12] C. Englund et al., "The grand cooperative driving challenge 2016: Boosting the introduction of cooperative automated vehicles," IEEE Wireles. Commun., vol. 23, no. 4, pp. 146-152, Aug. 2016.

[13] J. K. Hedrick, D. H. McMahnon, and D. Swaroop, "Vehicle modeling and control for automated highway systems," Univ. California, Berkeley, CA USA, Tech. Rep. UCB-ITS-PRR-93-24, 1993.

[14] V. Milanés, S. E. Shladover, J. Spring, C. Nowakowski, H. Kawazoe, and M. Nakamura, "Cooperative adaptive cruise control in real traffic situations," IEEE Trans. Intell. Transp. Syst., vol. 15, no. 1, pp. 296-305, Feb. 2014.

[15] L. Xiao, S. Darbha, and F. Gao, "Stability of string of adaptive cruise control vehicles with parasitic delays and lags," in Proc. 11th IEEE Int. Conf. Intell. Transp. Syst., Oct. 2008, pp. 1101-1106.

[16] S.-N. Huang and W. Ren, "Design of vehicle following control systems with actuator delays," Int. J. Syst. Sci., vol. 28, no. 2, pp. 145-151, 1997.

[17] F. Morbidi, P. Colaneri, and T. Stanger, "Decentralized optimal control of a car platoon with guaranteed string stability," in Proc. IEEE Eur. Control Conf., Jul. 2013, pp. 3494-3499.

[18] G. H. Golub and C. F. van Loan, Matrix Computations. Baltimore, MD USA: The Johns Hopkins Univ. Press, 1989.

[19] S. Öncü, J. Ploeg, N. van de Wouw, and H. Nijmeijer, "Cooperative adaptive cruise control: Network-aware analysis of string stability," IEEE Trans. Intell. Transp. Syst., vol. 15, no. 4, pp. 1527-1537, Aug. 2014.

[20] J. Ploeg, D. P. Shukla, N. van de Wouw, and H. Nijmeijer, "Controller synthesis for string stability of vehicle platoons," IEEE Trans. Intell. Transp. Syst., vol. 15, no. 2, pp. 854-865, Apr. 2014.

[21] J. K. Hedrick, M. Tomizuka, and P. Varaiya, "Control issues in automated highway systems," IEEE Control Syst., vol. 14, no. 6, pp. 21-32, Dec. 1994.

[22] C. Desjardins and B. Chaib-draa, "Cooperative adaptive cruise control: A reinforcement learning approach," IEEE Trans. Intell. Transp. Syst., vol. 12, no. 4, pp. 1248-1260, Dec. 2011. 
[23] S. Öncü, N. van de Wouw, and H. Nijmeijer, "Cooperative adaptive cruise control: Tradeoffs between control and network specifications," in Proc. Int. IEEE Conf. Intell. Transp. Systs., Oct. 2011, pp. 2051-2056.

[24] J. Ploeg, N. van de Wouw, and H. Nijmeijer, " $\mathcal{L}_{p}$ string stability of cascaded systems: Application to vehicle platooning," IEEE Trans. Control Syst. Technol., vol. 22, no. 2, pp. 786-793, Mar. 2014.

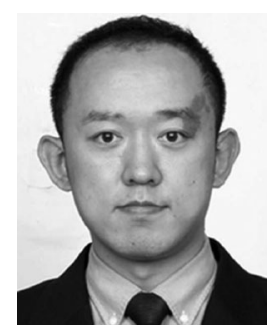

Haitao Xing received the B.Sc. and M.Sc. degrees in mechanical engineering from the College of Automotive Engineering, Jilin University, Changchun, China, in 2010 and 2013, respectively. He is currently working toward the Ph.D. degree in cooperative driving in the Department of Mechanical Engineering, Eindhoven University of Technology, Eindhoven, The Netherlands.

His interests focus on longitudinal controller design on cooperative adaptive cruise control systems considering the wireless communication delay and

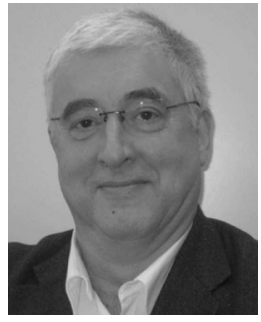

Henk Nijmeijer (M'83-SM'91-F'00) is a Full Professor at Eindhoven, and Chairs the Dynamics and Control group. He has published many journal and conference papers, and several books, and is or was on the Editorial Board of numerous journals.

Prof. Nijmeijer is the Editor of Communications in Nonlinear Science and Numerical Simulations. He was appointed Honorary Knight of the Golden Feedback Loop in 2011. Since 2011, he has been an IFAC Council Member. Since January 2015, he is the Scientific Director of the Dutch Institute of Systems and Control, The Netherlands. He received the IEE Heaviside Premium Award in 1990. He received the 2015 IEEE Control Systems Technology Award.

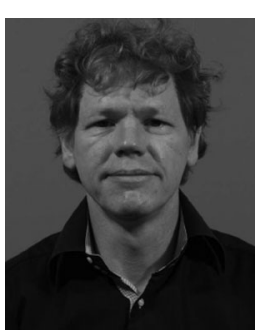

Jeroen Ploeg received the M.Sc. degree in mechanical engineering from the Delft University of Technology, Delft, The Netherlands, in 1988, and the Ph.D degree in mechanical engineering on the control of vehicle platoons from the Eindhoven University of Technology, Eindhoven, The Netherlands, in 2014.

From 1989 to 1999, he was affiliated with Koninklijke Hoogovens (currently Tata Steel), IJmuiden, The Netherlands, where his interest was the development and implementation of dynamic process control systems for large-scale industrial plants. Since 1999, he has been a Senior Research Scientist with the Integrated Vehicle Safety Department, TNO, Helmond, The Netherlands. His interests include control system design for cooperative and automated vehicles in general and automated vehicle platoons in particular and motion control of wheeled mobile robots. This research is executed in close cooperation with the Department of Mechanical Engineering, Eindhoven University of Technology. Dr. Ploeg is currently an Associate Editor for the IEEE TRANSACTIONS ON INTELLIGENT TRANSPORTATION SYSTEMS. 